\title{
Anodic-oxide-induced interdiffusion in quantum wells structure
}

\author{
M. C. Y. Chan and E. Herbert Li \\ Department of Electrical and Electronic Engineering, University of Hong Kong, Pokfulam Road, Hong Kong \\ Tel: (852) 2859-7091, Fax: (852) 2559-8738, Email: ehli@eee.hku.hk \\ Shu Yuan, H. H. Tan, and C. Jagadish \\ Department of Electronic Materials Engineering, Research School of Physical Sciences and Engineering, The \\ Australian National University, Canberra, ACT 0200, Australia
}

\begin{abstract}
Enhancement of interdiffusion in GaAs/AlGaAs quantum wells (QWs) due to anodic oxides was studied. Photoluminescence and diffused QW modeling were used to understand the effects of intermixing on the QW structure. The activation energy is similar to those obtained from $\mathrm{SiO}_{2}$ cap annealed quantum well structures.
\end{abstract}

\section{INTRODUCTION}

Interdiffusion in quantum wells (QW) has recently attracted much interest in device applications and physics [1-3], since it can be used to modify materials and device properties. Interdiffusion between QW and adjacent barriers results in a change in QW shape which in turn modifies the subband energies in the conduction band and the valence band [4]. As a result, the interband transition energy is modified after growth without etching or epitaxial re-growth, which can be used to post-growth modify the lasing wavelength of QW lasers and the absorption coefficient of waveguides and photodetectors, and to improve lateral carrier confinement of ridge waveguide lasers.

Recently we have demonstrated a novel impurity-free interdiffusion in GaAs/AlGaAs system [5], namely anodic-oxide-induced interdiffusion. A portion of the GaAs cap layer of the GaAs/AlGaAs quantum well structure is anodically oxidized, the oxide enhances the interdiffusion at high temperature. The anodic-oxide-induced interdiffusion is very simple: the anodization is done at room temperature for a few minutes without special sample preparation, the RTA is done in a flowing gas rapid thermal annealing system very conveniently. Selective area interdiffusion can be done easily as the surface of the sample can be easily patterned before anodization. This interdiffusion technique can be conveniently applied to the fabrication of nonabsorbing mirrors for high power laser application and to the fabrication of multiple wavelength lasers. In this paper, we will describe in detail the anodic-oxide-induced interdiffusion in GaAs/AlGaAs quantum structures.

\section{SAMPLES AND EXPERIMENTS}

The structure (Sample A) is grown by low pressure metal organic chemical vapor deposition (MOCVD) on $\mathrm{p}^{+}$-GaAs substrates. The substrates are $\mathrm{Zn}$-doped at $1 \times 10^{19} \mathrm{~cm}^{-3}$ concentration and $2^{\circ}$ off $<100\rangle$ towards $\langle 110\rangle$. The layer structures for these samples are listed in Tab. I. All epilayers are nominally undoped.

Table I. Samples structure. All epilayers are nominally
undoped and grown on the $\mathrm{P}^{+}$GaAs substrates by low pressure
MOCVD.
\begin{tabular}{|c|c|}
\hline \multicolumn{2}{|c|}{ Sample A } \\
\hline GaAs & $300 \mathrm{~nm}$ \\
\hline $\mathrm{Al}_{0.3 \mathrm{Ga} 0.7 \mathrm{As}}$ & $50 \mathrm{~nm}$ \\
\hline $\mathrm{GaAs}(\mathrm{QW})$ & $3.2 \mathrm{~nm}$ \\
\hline $\mathrm{Al}_{0.3 \mathrm{Ga} 0.7 \mathrm{As}}$ & $50 \mathrm{~nm}$ \\
\hline $\mathrm{GaAs}(\mathrm{buffer})$ & $500 \mathrm{~nm}$ \\
\hline $\mathrm{P}^{+} \mathrm{GaAs}$ & substrate \\
\hline
\end{tabular}

After growth; samples of area of $8 \mathrm{~mm} \times 8 \mathrm{~mm}$ are cleaved. Half of each sample was then covered with thermal glue. Each sample is rinsed in $10 \% \mathrm{HCl}$ and de-ionized water, and nitrogen blow dried before it is picked up on the backside by a vacuum tweezers made by a stainless steel tube as shown in Fig. 1. The sample is then lowered to touch the electrolyte and then pulled a little bit up. The electrolyte is composed of ethylene glycol: de-ionized water: phosphoric acid (40:20:1 by volume). The anodization is performed at room temperature. The pulse width is $1.0 \mathrm{~ms}$, the period is $12.0 \mathrm{~ms}$, the total pulsed anodization time is 4 minutes. The pulsed anodization current density is in the range of 40 to $160 \mathrm{~mA} / \mathrm{cm}^{2}$ as determined by the leading edge of the pulse. The area covered with thermal glue is unanodized, while anodization takes place in the uncovered area to form a native oxide layer. When the pulse is on, anodization takes place, and when the pulse is off, the anodic oxide is etched slowly by the electrolyte. After anodization, samples are rinsed with de-ionized water, followed by acetone to remove the 
thermal glue, then by de-ionized water and $\mathrm{N}_{2}$ blow dried. Each sample is then cleaved into two $4 \mathrm{~mm} \times 8$ $\mathrm{mm}$ pieces, so that each piece has half area anodized ( 4 $\mathrm{mm} \times 4 \mathrm{~mm}$ ) and half area unanodized (4 $\mathrm{mm} \times 4$ $\mathrm{mm})$. This enables a reasonable TEM and PL comparison between anodized and unanodized areas after interdiffusion process. The interdiffusion is carried out in a RTA chamber with GaAs proximity cap in flowing argon ambient, the temperature ramp rate is $50 \mathrm{C} / \mathrm{s}$.

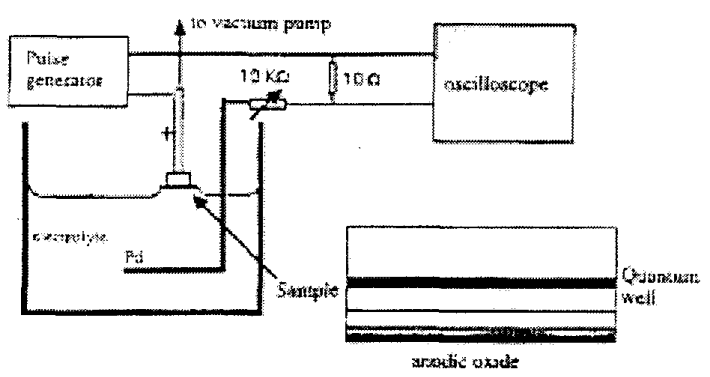

FIG. 1. Schematic diagram showing the anodic oxidization set-up used in this work. The electrolyte is composed of ethylene glycol, de-ionized water, and phosphoric acid (40:20:1 by volume). The pulse generator provides voltage pulses of $1 \mathrm{~ms}$ width and $12 \mathrm{~ms}$ period. The anodization time was usually 4 minutes.

After the interdiffusion process, TEM is used to determine these quantum well structures [5]. A technique is developed to determine the $\mathrm{Al}$ profile of the quantum wells from the TEM images. $90^{\circ}$-wedge TEM specimens are prepared by the cleavage method. Investigations of these specimens are performed in a Philips EM-430 transmission electron microscope operating at $300 \mathrm{kV}$. In the experiment, the specimens are orientated to the [100] zone axis and bright-field images are recorded using a CCD camera with a Gatan digital imaging software. Al profiles are determined by comparing the thickness-fringes in experimental images with profiles calculated for different $\mathrm{Al}$ contents at $5 \%$ steps. This technique will be published elsewhere in detail.

Low temperature (12K) PL measurements are performed on both the anodic oxidized and unoxidized sides of the samples. PL excitation is by an Argon ion laser $(\lambda=514.5 \mathrm{~nm})$ modulated by a mechanical chopper. Excitation power is about $5 \mathrm{~mW}(\mathrm{CW})$. The detection system consisted of a $0.75 \mathrm{~m}$ grating spectrometer, Silicon photo detector, and a lock-in amplifier referenced to the mechanical chopper. Spectra are acquired by a personal computer. A few samples are mounted together on the same cold finger in a cryostat, so that the PL intensity can be reasonably compared to each other. For anodized samples without RTA treatment, the PL energies do not show significant shift from original as-grown sample.

\section{MODELLING OF THE INTERDIFFUSION}

In order to determine the interdiffusion parameters, such as diffusion coefficient and activation energy, from the experimental data, we first calculate the subband energies and wavefunctions in an interdiffusion modified single non-square quantum well [4], and then compare the calculated interband transition energy to the PL data. The confinement profile of this interdiffused quantum well is non-linear and is modelled here by an error function.

The extent of the disordering process is characterized by a diffusion length $\mathrm{L}_{d}$, which is defined here as $L_{d}=\sqrt{D t}$, where $D$ and $t$ are the diffusion coefficient and annealing time, respectively. In the model presented here we assume an isotropic interdiffusion of $\mathrm{Al}$ and $\mathrm{Ga}$ with the diffusion coefficients being independent of their respective concentrations, which are also assumed to be equal and constant, i.e. according to Fick's second law of diffusion [6]. The diffused Al composition profile, $\widetilde{W}(z)$, across the $Q W$ structure is given by:

$$
w(z)=w_{o}\left\{1-\frac{1}{2}\left[\operatorname{erf}\left(\frac{L_{z}+2 z}{4 L_{d}}\right)+\operatorname{erf}\left(\frac{L_{z}-2 z}{4 L_{d}}\right)\right]\right\}(1)
$$

where $W_{0}$ is the as-grown $\mathrm{Al}$ mole fraction in the barrier, $L_{z}$ is the as-grown width of the $\mathrm{QW}, \mathrm{z}$ is both the quantization and the growth axis (QW centered at $z=0$ ), and erf denotes the error function [7]. The calculated quantum well interband exciton transition energies are fitted to the measured of energy shift of PL peak by adjusting the value of $D$. The diffusion coefficient $\mathrm{D}$ can also be expressed by the Arrhenius relationship

$$
\mathrm{D}=\mathrm{D}_{0} \mathrm{e}^{-\mathrm{E}_{\mathrm{A}} / \mathrm{kT}}
$$

where $D_{0}$ is a prefactor, $E_{A}$ is the activation energy, $k$ is the Boltzmann constant, and $T$ is the annealing temperature. $\mathrm{D}_{0}$ and $\mathrm{E}_{\mathrm{A}}$ are determined by a linear fit to the resulting values of $\operatorname{lnD}$ versus $1 / \mathrm{kT}$. The $\mathrm{Al}$ profiles for various quantum wells are also calculated by the Eq. (1) using this fitted value of $D$.

\section{RESULTS AND DISCUSSION}

Fig. 2 shows PL spectra from Sample-A. The samples are annealed at $875 \mathrm{C}, 900 \mathrm{C}, 925 \mathrm{C}$, and $950 \mathrm{C}$, the annealing time is $120 \mathrm{~s}$. The upper part are the spectra from those unanodized areas, while the lower part are the spectra from the anodized areas. The unanodized quantum well is relatively thermally stable up to $925 \mathrm{C}$, then it starts to show a strong blueshift at 950C. PL from anodized samples show continuous blue shift and significant intensity increase for annealing temperatures above $925 \mathrm{C}$. This trend is observed for all samples anodized at different current densities. No linewidth broadening is observed from the PL spectra of the anodic-oxide-induced interdiffused samples. 


\section{CONCLUSIONS}

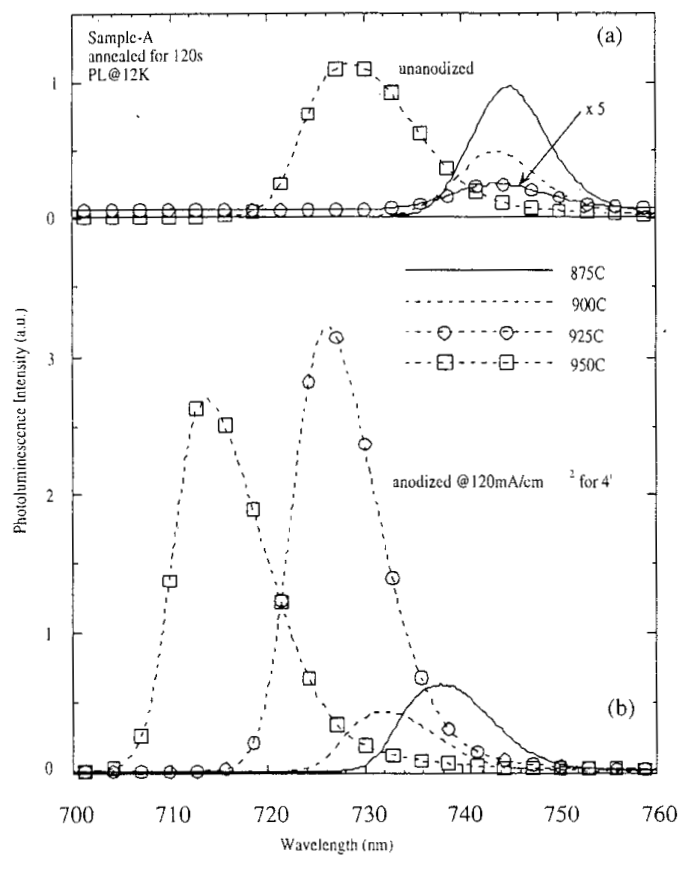

FIG. 2. Photoluminescence spectra at $12 \mathrm{~K}$ for Sample-A annealed at various temperatures for 120 s with (lower part) and without (upper part) anodic oxide. The anodization current density is $120 \mathrm{~mA} / \mathrm{cm}^{2}$ and the total anodization time is 4 minutes for all structures.

In Fig. 3, the PL peak energy shift of samples from Sample- $A$ is plotted against annealing temperature for various anodization current densities. Also shown is the data for unanodized but annealed control samples of Sample-A as a control reference. The annealing time is $120 \mathrm{~s}$. The anodic oxide induced interdiffusion is not sensitive to the anodic current density in the range from $40 \mathrm{~mA}^{-\mathrm{cm}^{2}}$ to $160 \mathrm{~mA} / \mathrm{cm}^{2}$ in the temperature range studied. This is due to the fact that the anodic oxide thickness is not sensitive to the anodization current density. The anodic oxide thickness' for samples of Sample-A anodized at 80 , 160 , and $200 \mathrm{~mA} / \mathrm{cm}^{2}$ for 4 minutes are determined to be 125,110 , and $125 \mathrm{~nm}$, respectively. The diffusion coefficients $D$ can be determined by Eq. (2). For the $120 \mathrm{~mA} / \mathrm{cm}^{2}$ anodized and interdiffused samples of Sample-A used in Figs. $2-3, D_{0}=1.12 \times 10^{-3} \mathrm{~cm}^{2} / \mathrm{s}$ and $\mathrm{E}_{\mathrm{A}}=3.19 \mathrm{eV}$. The diffusion coefficients are plotted in Fig. 4 together with a fit. The diffusion coefficient is somehow smaller than those obtained by $\mathrm{SiO}_{2}$ impurity-free induced interdiffusion, but the activation energy is similar [8,9]. In this study, the anodic-oxide thickness is about $120 \mathrm{~nm}$, if it is increased (say, to $200 \mathrm{~nm}$ ), larger interdiffusion effect can be expected.
In conclusion, we have reported a new method for creation of interdiffused quantum wells. This technique is simple and inexpensive, and can be easily adopted in opto-electronic device fabrication. Current density used during formation of anodic oxides has very little influence on the interdiffusion process. The activation energy is similar to those obtained from $\mathrm{SiO}_{2}$ cap annealed quantum well structures.

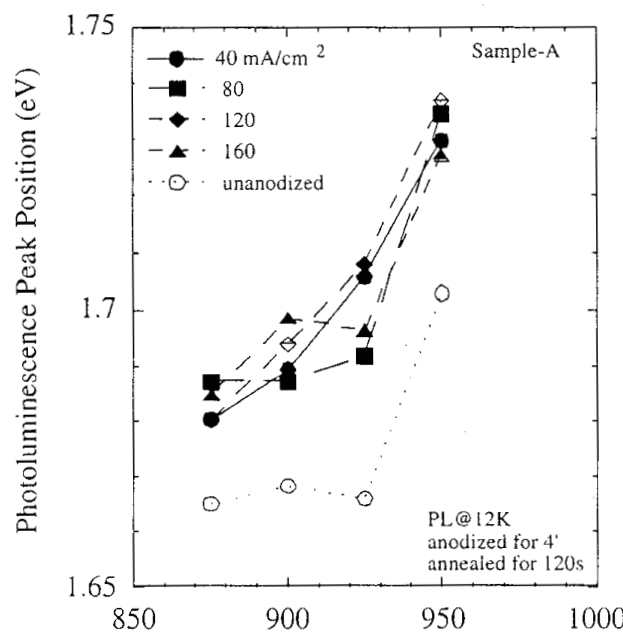

Rapid Thermal Annealing Temperature (C)

FIG. 3. Photoluminescence peak position as a function of rapid themal annealing temperature for various anodic oxidization current densities. Lines are only shown to connect the experimental points.

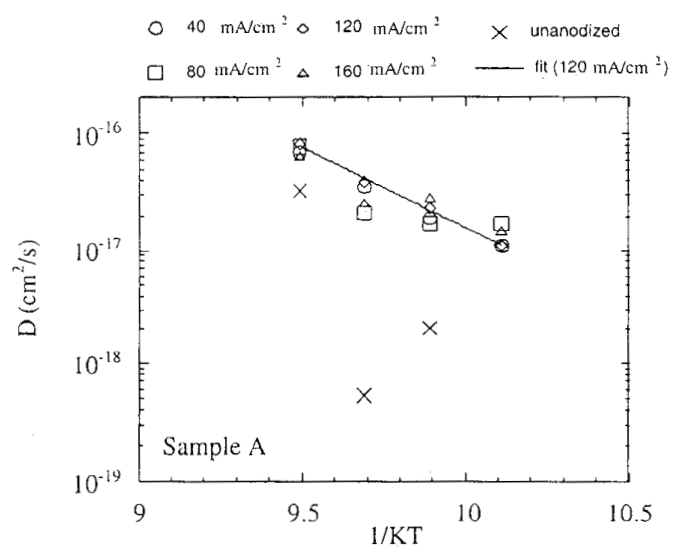

FIG. 4. Plot of $D$ as a function of $(1 / \mathrm{KT})$ for Sample-A annealed for 120s. The experimental data were fitted by the Arrhenius relationship. 


\section{ACKNOWLEDGMENTS}

This work is partly supported by the RGC earmarked grant of Hong Kong, the University of Hong Kong CRCG grant, and partly by Australian Research Council.

\section{REFERENCES}

[1] E. H. Li, Ed, Quantum Well Intermixing for Photonics, (SPIE Milestone Series, Bellingham 1997)

[2] D. G. Deppe and H. Holonyak, Jr., "Atom diffusion and impurity-induced layer disordering in quantum well III-V semiconductor heterostructures",J. Appl. Phys., vol. 64, pp. R93-113, 1988.

[3] J. H. Marsh, "Quantum well intermixing", Semicond. Sci. Technol., vol. 8, pp. 1136-1155, 1993.

[4] E. H. Li, B. L. Weiss, and K. S. Chan, "Effect of interdiffusion on the subbands in an $\mathrm{Al}_{\mathrm{x}} \mathrm{Ga}_{1}$. xAs/GaAs single-quantum-well structure", Phys. Rev. B, vol.46, pp. 15181-15192, 1992.
[5] S. Yuan, Y. Kim, C. Jagadish, P. T. Burke, M. Gal, J. Zou, D. Q. Cai, D. J. H. Cockayne, and R. M. Cohen, "Novel impurity-free interdiffusion in GaAs/AlGaAs quantum-wells by anodization and rapid thermal annealing", Appl. Phys. Lett., vol. 70, pp. 1269-1271, 1997.

[6] J. Crank, The Mathematics of Diffusion. 2nd edition. Oxford : Oxford University, 1975, p.15.

[7] M. Abramowitz and I. A. Stegun, Handbook of Mathematical Functions. Washington, D.C. : National Bureau of Standards, 1964.

[8] S. Burkner, M. Maier, E. C. Larkins, W. Rothemound, E. P. O'Reilly, and J. D. Ralston, "Process parameter dependence of impurity free interdiffusion in $\mathrm{GaAs} / \mathrm{Al}_{x} \mathrm{Ga}_{1-x} \mathrm{As}$ and $\mathrm{In}_{\mathrm{y}} \mathrm{Ga}_{1 \text {. }}$ y As/GaAs multiple quantum wells, J. Electronic Materials, vol. 24, pp. 805-812, 1995.

[9] I. Gontijo, T. Krauss, J. H. Marsh, and R. M. De La Rue, "Postgrowth control of GaAs/AlGaAs quantum well shapes by impurity-free vancancy diffusion", IEEE J. Quantum Electron., vol. 30, pp. 1189-1194, 1994. 